\title{
Musculoskeletal Simulation for Assessment of Effect of Movement-Based Structure-Modifying Treatment Strategies
}

\author{
Tien Tuan Dao \\ Sorbonne Universités, Université de Technologie de Compiègne, CNRS, UMR 7338, Biomécanique et Bioingénierie, \\ Centre de Recherche Royallieu, CS 60 319, 60203 Compiègne, France
}

Correspondence should be addressed to Tien Tuan Dao; tien-tuan.dao@utc.fr

Received 6 August 2015; Revised 2 December 2015; Accepted 7 December 2015

Academic Editor: Camillo Porcaro

Copyright (C) 2015 Tien Tuan Dao. This is an open access article distributed under the Creative Commons Attribution License, which permits unrestricted use, distribution, and reproduction in any medium, provided the original work is properly cited.

The better understanding of the complex mechanism between neural motor control and its resulting joint kinematics and muscle forces allows a better elucidation of the mechanisms behind body growth, aging progression, and disease development. This study aimed at investigating the impact of movement-based structure-modifying treatment strategies on joint kinematics, muscle forces, and muscle synergies of the gait with instrumented implant. A patient-specific musculoskeletal model was used to quantitatively assess the deviations of joint and muscle behaviors between the normal gait and 4 gait modifications (bouncy, medial thrust, midcrouch, and mtp (i.e., gait with forefoot strike)). Moreover, muscle synergy analysis was performed using EMG-based nonnegative matrix factorization. Large variation of 19 degrees and $190 \mathrm{~N}$ was found for knee flexion/extension and lower limb muscle forces, respectively. EMG-based muscle synergy analysis revealed that the activation levels of the vastus lateralis and tibialis anterior are dominant for the midcrouch gait. In addition, an important contribution of semimembranosus to the medial thrust and midcrouch gaits was also observed. In fact, such useful information could allow a better understanding of the joint function and muscle synergy strategies leading to deeper knowledge of joint and muscle mechanisms related to neural voluntary motor commands.

\section{Introduction}

Accurate simulation of the human dynamics movement is one of the most challenging research topics in the last two decades in the biomechanics field. Most of the research projects focused on the in vivo estimation of joint kinematics and muscle forces during motion [1-6]. Such information could be of great clinical interest to objectively evaluate the mechanical functions of the musculoskeletal system in normal as well as in pathological conditions [7-9]. In the case of orthopedic device prescription especially, the joint kinematics and muscle force information could allow an optimal design to be performed leading to maximizing the benefit of the involved patient [10, 11]. In addition, structure-modifying treatment modalities such as voluntary gait modification strategies have been studied to alter the medial knee joint load leading to decreasing the external knee adduction moment (KAM) [12-14]. This conservative nonpharmacologic management allows symptoms or disease progression to be alleviated for degenerative joint disorders such as osteoarthritis (OA).

A recent review study [15] showed kinetic and kinematic changes by using some gait modifications such as increases of step width or cadence or knee flexion or mediolateral trunk lean or weight transfer to the medial foot or hip internal rotation or toe-out or speed as well as reductions of vertical acceleration and initial contact or stride length or toeoff or speed or using gait aids (e.g., ipsilateral/contralateral cane) and applications of specific gait patterns (medial knee thrust or Tai Chi gait). These gait modifications have shown their efficiencies in the management of knee joint disease. In fact, the use of contralateral cane and the increase of trunk lean lead to reducing the early-stance KAM. Besides, the increase of toe-out modification leads to reducing the latestance KAM. Particularly, the medial knee thrust gait altered dynamically knee alignment and thus KAM lever arm leading to the reduction of KAM during gait cycle. Despite their great potentials, further investigation is needed to elucidate 
TABLE 1: Description of normal gait and gait modification strategies.

\begin{tabular}{|c|c|c|c|}
\hline Gait modifications & Description & Marker's number & $\begin{array}{c}\text { Trial name: gait cycle times (start > end) } \\
\text { (time in sec) }\end{array}$ \\
\hline Ngait & Normal gait & 56 & Ngait_og1: $1.699>2.811$ (1.112) \\
\hline Bouncy & $\begin{array}{l}\text { Increased superior-inferior } \\
\text { translation of the pelvis }\end{array}$ & 52 & Bouncyl: $2.066>3.214(1.148)$ \\
\hline Medial thrust & $\begin{array}{l}\text { Internally rotated hip of the stance } \\
\text { leg }\end{array}$ & 52 & Medthrust2: 2.415 > 3.571 (1.156) \\
\hline Midcrouch & $\begin{array}{l}\text { Crouched position with a mild } \\
\text { increase in knee flexion angle }\end{array}$ & 56 & Mildcrouch1: $10.822>11.901$ (1.079) \\
\hline Mtp & Gait with forefoot strike & 52 & Mtpgait2: $2.276>3.421(1.145)$ \\
\hline
\end{tabular}

the effect of voluntary gait modifications on the muscle force, which is still not fully understood.

There exist many cutting-edge computational approaches to estimate the joint kinematics and muscle force. The first one related to inverse dynamics and static optimization [4, 16]. This approach used skin-mounted marker's trajectories and ground reaction forces within a musculoskeletal model. The second approach deals with the EMG-driven forward dynamic. In this case, the surface electromyography is commonly used to define the muscle activation pattern [17] to reproduce the observed motion. The third approach is the dynamic optimization using muscle activation-driven pattern to generate the body motion $[18,19]$. This approach is computationally complex, but the results are practically equivalent to the static optimization [20]. The choice of the appropriate approach depends on the specific objective as well as on the availability of input data. However, the interaction between neural motor command and musculoskeletal structures remains a challenging issue. One of the current most-used strategies related to the replacement of the neural motor command by the hypothesis of the performance strategies of the human body. In fact, the optimization of muscle metabolic function is one of the most used hypotheses when using inverse dynamics and static optimization approach. Thus, the understanding of this underlying interaction could allow a deeper knowledge of the mechanical function of the human body, especially in the cases of muscle weakness or spasticity and muscle synergies.

Motor cortex is involved in planning, control, and execution of voluntary motions. The motor cortex gradually performs the coordination of several muscles in the control of body forces and motions when the human being learns a new complex locomotion pattern. The understanding of this complex coordination mechanism may be elucidated by brain-based electrical stimulation [21,22]. Moreover, Barroso et al. [23] studied the muscle coordination strategy by muscle synergy analysis during walking and running. In fact, muscle synergies relate to the coordinated and temporal recruitment of muscle groups to perform a specific task. However, muscle synergies have not yet been compared between the normal gait and voluntary gait modification patterns. The question of how the module function (i.e., muscle synergy) changes from the normal gait to other voluntary altered gaits is still misunderstood. The response to this question may provide some elucidation for robotic application using neural control strategies or for testing and optimizing the implant effect on the human locomotor function. In fact, the objectives of this present study were to (1) quantitatively assess the effect of voluntary neural command through 4 altered gait modifications (bouncy, medial thrust, midcrouch, and mtp (i.e., gait with forefoot strike)) on the joint kinematics and muscle force estimation and (2) find muscle synergy patterns related to the normal gait as well as these altered gait modifications.

\section{Materials and Methods}

2.1. Patient Data. The patient data used in this present study were extracted from the fourth edition of the Knee Grand Challenge [24]. All used data are briefly summarised here. Data acquisition was performed on a patient (male, 88 years old, $168 \mathrm{~cm}$ body height, $66.7 \mathrm{~kg}$ body weight, and $23.6 \mathrm{~kg} / \mathrm{m}^{2}$ Body Mass Index (BMI)) with knee implant instrumented in his right knee side. The patient had Rockport flat bottom sneakers shoes during the gait data acquisition process. The patient performed an overground normal gait pattern, which was used as reference pattern. Then, 4 overground gait trials reflecting 4 gait modification strategies (bouncy, medial thrust, mild crouch, and mtp (i.e., gait with forefoot strike)) were acquired using an 8-camera Vicon system. All skin-mounted markers were posed using a modified Cleveland Clinic marker protocol [24]. Kinematical sampling frequency is $120 \mathrm{~Hz}$. Ground reaction forces were acquired using 3 AMTI force plates. Sampling frequency of the ground reaction forces is $1000 \mathrm{~Hz}$. Moreover, during each acquisition trial, surface electromyography (EMG) of 15 lower limbs muscles (semimembranosus, biceps femoris, vastus medialis, vastus lateralis, rectus femoris, medial gastrocnemius, lateral gastrocnemius, tensor fascia latae, tibialis anterior, peroneus longus, soleus, adductor magnus, gluteus maximus, gluteus medius, and sartorius) was also monitored using Delsys Bagnoli EMG System. The description of the normal gait and these gait modification strategies is depicted in Table 1. Furthermore, pre- and postsurgery CT data were also acquired on the patient using a Siemens CT machine; the slice thickness was set up as $0.6 \mathrm{~mm}$. The matrix is $512 \times 512$ and the pixel resolution is $0.9 \times 0.9 \mathrm{~mm}^{2}$. The number of slices is 1900 for the whole scanned lower limb. 


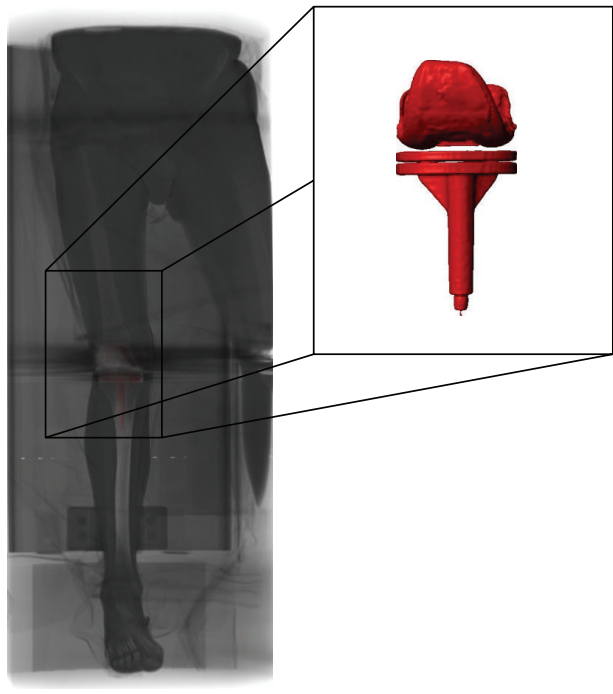

(a)

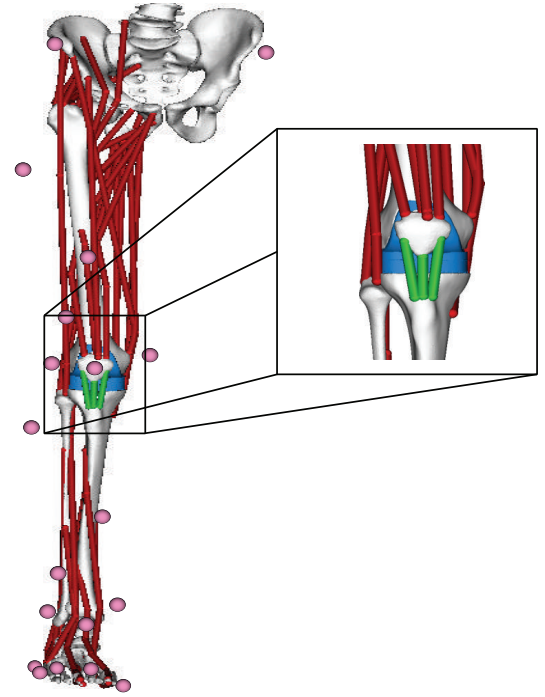

(b)

FIGURE 1: Patient-specific rigid musculoskeletal model: CT-based visualization of the lower limb with implant (a) and its respective OpenSIM model (b).

2.2. Patient-Specific Rigid Musculoskeletal Model. An available 7-segment patient-specific OpenSIM musculoskeletal model was utilized to compute gait kinematics and muscle forces for normal gait and gait modification patterns [25] (Figure 1). The model composed of the pelvis and right leg of the patient under investigation. Post- and presurgery CT data and laser-scan-based implant component were used to develop geometric model. The model development process is briefly described here. Image segmentation using SliceOmatic (Montreal, Canada) was performed to extract bony segments (pelvis, femur, patella, tibia, fibula, talus, calcaneus, and phalanges) and implant components (femoral component, tibial tray). Data from postsurgery (spanned from the proximal end of the pelvis to the distal tips of the toes) was aligned with those from presurgery data (approximately $15 \mathrm{~cm}$ above and below the joint line). STLbased surface geometries were created and imported into OpenSIM to develop corresponding bony segments. The model has 24 degrees of freedom (DOF) (6 DOF groundto-pelvis joint, 3 DOF hip joint, 6 DOF tibiofemoral joint, 6 DOF patellofemoral joint, 2 DOF ankle joint, and 1 DOF toes joint). The interaction between implant components and its respective bone was modeled using a weld joint. The interaction between implant components (femoral component, tibial tray, and patella/femoral component) was modeled using a reverse joint. All segment coordinate systems were based on the ISB recommendations [26]. The model has 22skin-mounted virtual marker's cluster according to the real market cloud used in the motion capture acquisition. Mass and inertial properties of segmental bodies were scaled using the data from Arnold et al. [27].

44 lower limb muscles were integrated into the bony model. Each muscle was modeled as a Schutte muscle model [28]. Muscle attachment points and wrapping surface were created using an available lower limb model [27].
A transformation process using information from bone-tobone alignment was performed to morph the patient-specific musculature. Some attachments' points and unrealistic intersection of muscle lines of action were manually adjusted.

2.3. Estimation of Joint Kinematics and Muscle Forces. Based on skin-mounted marker's trajectories, inverse kinematics was performed to compute the joint kinematics. Then, inverse dynamics and static optimization $[2,4,16]$ were applied to estimate the muscle forces according to the normal gait and each gait modification pattern. All these algorithms were performed using OpenSIM 3.1 version on a Dell computer (Precision T3500, 2.8 Ghz, $3 \mathrm{~GB}$ RAM). Inverse dynamic aims at estimating net joint moments from tracked segment kinematics. Static optimization allows the estimation of the muscle forces to be performed using the equilibrium principle between net joint moments, muscle lever arms, and muscle forces. In this present study, the optimization problem is expressed as follows:

$$
\begin{aligned}
\operatorname{minimize} & F_{\mathrm{obj}} \\
\text { subject to } & R(q) F^{T M}=T_{M T} \\
& 0 \leq F^{T M} \leq F_{M}^{0},
\end{aligned}
$$

where $q$ is the joint angles set for $n$ joints; $R(q)$ is the muscle moment arms $(n \times m)$ matrix; $F^{T M}$ are the muscle force $(m \times 1)$ matrix; $T_{M T}$ is the muscular joint moments $(n \times 1)$ matrix; $F_{M}^{0}$ is the peak isometric muscle force deriving from the crosssectional area of the muscle; and $F_{\text {obj }}$ is an activation-based objective function as follows:

$$
F_{\mathrm{obj}}=\left(\sum_{j=1}^{N} a_{j}\left(t_{i}\right)\right)^{2},
$$




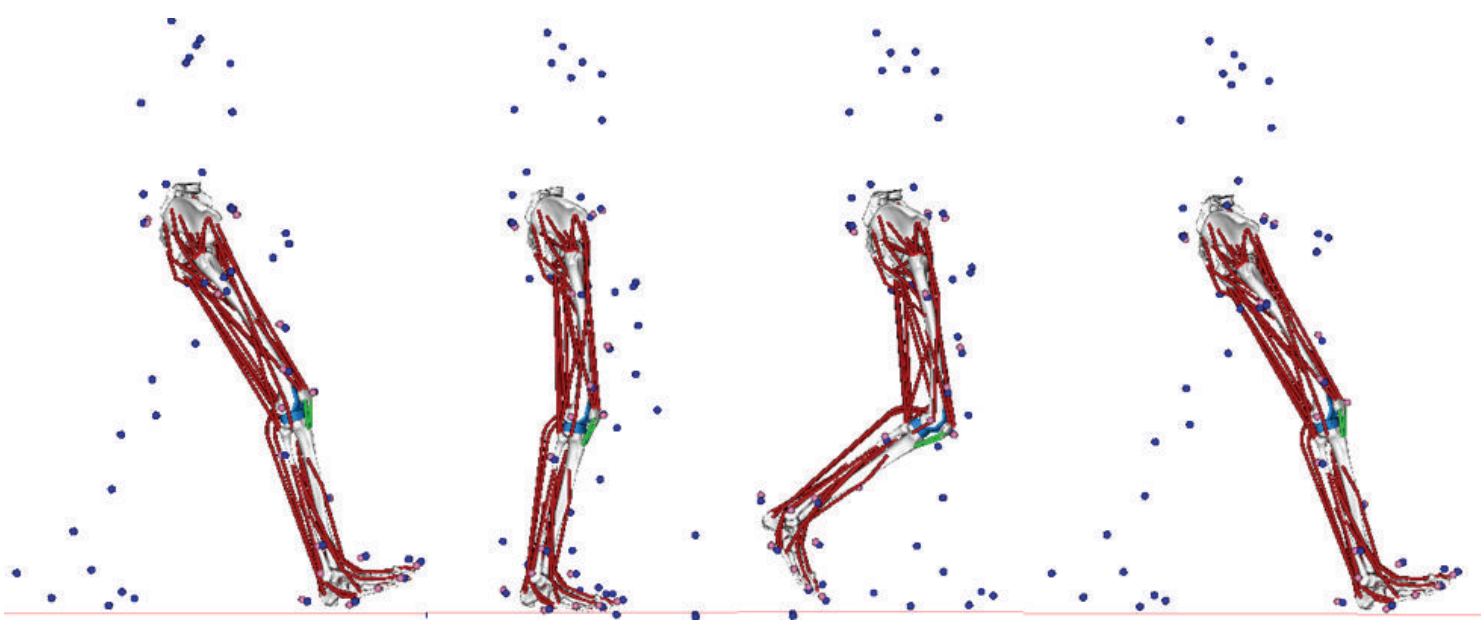

FIGURE 2: Visualization of the normal gait motion of the patient-specific model superposed with the experimental 56-skin-mounted (blue color) and virtual 22-skin-mounted (pink color) marker clusters.

where $a_{j}$ is the activation level of the muscle $j$ and $N(=44)$ is the number of muscles of interest.

To assess the kinematical deviation, a peak-to-peak absolute error was computed for each pair of comparison between the normal gait pattern and each gait modification pattern. The deviation of muscle forces was quantified using root mean square error (RMSE) and its relative error (RE) according to the maximal value. These quantities were calculated for each pair of comparison between the normal gait pattern and each gait modification pattern. Respective mathematical equations of these errors are expressed as follows:

$$
\begin{aligned}
\mathrm{RMSE} & =\sqrt{\frac{\sum_{i}\left(X_{i}-Y_{i}\right)^{2}}{N},} \\
\mathrm{RE} & =\frac{\mathrm{RMSE}}{\max \left(\max \left(X_{i}\right), \max \left(Y_{i}\right)\right)},
\end{aligned}
$$

where $X_{i}$ and $Y_{i}(i \in\{1, \ldots, N\})$ are time series of two data sets used for the comparison. All postprocessing steps were performed using MATLAB R2010b (MathWorks, USA).

2.4. Muscle Synergy Analysis. The muscle synergies during the normal gait and respective gait modifications were computed using nonnegative matrix factorization method [2931]. This multivariate analysis and linear algebra tool allows transforming the EMG signals into muscle synergy features in reducing their dimensionality [32]. The respective equation of this feature transformation is expressed as follows:

$$
\mathrm{EMG}_{n m}=W_{n p} \times H_{p m}+R_{m}
$$

where $\mathrm{EMG}_{n m}$ is the matrix of EMG signals of $m$ muscles; $W_{n p}$ is the synergy matrix in which vector columns correspond to relative muscle activation levels; $p$ is the number of synergies; $n$ is the number of time steps; $H_{p m}$ is the synergy activation vector reflecting the EMG-to-force behavior; and $R_{m}$ is the factorization residual vector. The nonnegative matrix factorization method was implemented using MATLAB R2010b (MathWorks, USA).

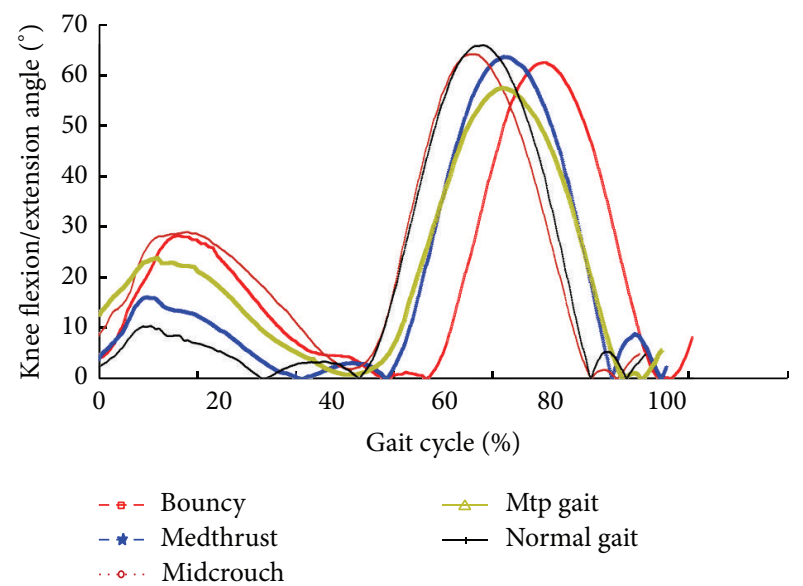

FIGURE 3: Evolution of the patient right knee flexion/extension within his instrumented implant during normal gait and altered gait modifications.

\section{Results}

3.1. Kinematical Deviation. A motion of the patient-specific model within the knee implant during his normal gait cycle is shown in Figure 2. The duration of his normal gait cycle is around $1.112 \mathrm{sec}$. The time durations of the bouncy, medial thrust, midcrouch, and mtp (i.e., gait with forefoot strike) gaits are around 1.148, 1.156, 1.079, and $1.145 \mathrm{sec}$, respectively. The evolutions of the knee flexion/extension within his instrumented implant during normal and modified gaits are illustrated in Figure 3. The 1st and 2nd peak-to-peak deviations of the knee flexion/extension motion of each modified gait according to the normal gait are depicted in Table 2. The 1st peak-to-peak deviation showed an increase of flexion/extension angle ranging from 5.6 (medial thrust) to 18.5 (midcrouch) degrees for all comparisons. The 2 nd peakto-peak deviation showed a decrease of flexion/extension angle ranging from 1.8 (midcrouch) to 8.52 (mtp) degrees for all comparisons. It is important to emphasize that, due to 

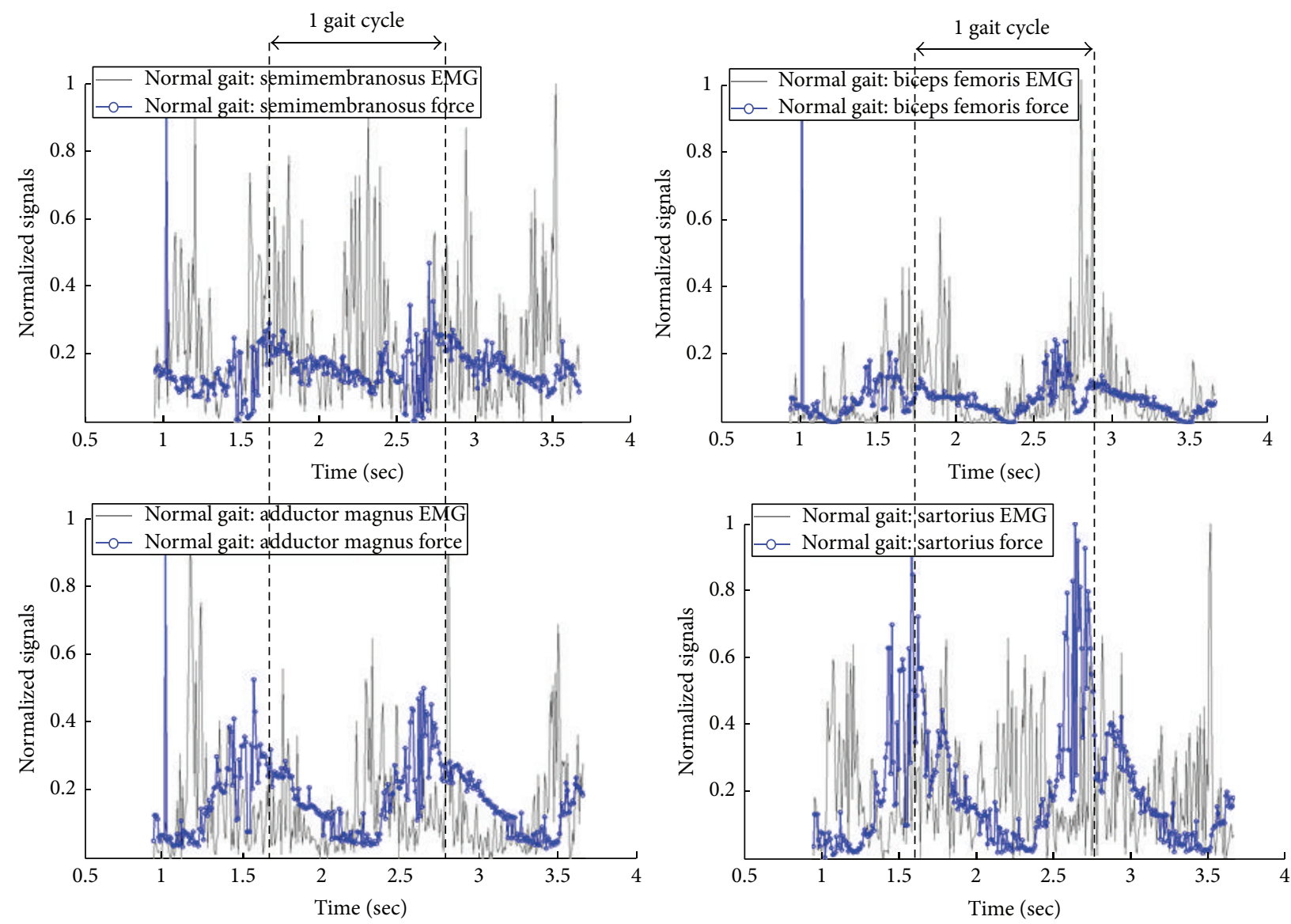

FIGURE 4: Lower limb muscle behaviors during the normal gait: normalized tensile forces versus EMG signals.

TABLe 2: Kinematical deviations between the normal gait and modification gait patterns.

\begin{tabular}{lcc}
\hline & $\begin{array}{c}\text { 1st peak-to-peak } \\
\text { deviation }\left(^{\circ}\right)\end{array}$ & $\begin{array}{c}\text { 2nd peak-to-peak } \\
\text { deviation }\left(^{\circ}\right)\end{array}$ \\
\hline Bouncy versus normal gait & 17.7 & -3.44 \\
Medthrust versus normal gait & 5.6 & -2.42 \\
Midcrouch versus normal gait & 18.5 & -1.8 \\
Mtpgait versus normal gait & 13.4 & -8.52 \\
\hline
\end{tabular}

the instrumented knee implant, these gait patterns fall out of normality with some cusps effects according to conventional gait patterns.

\subsection{Muscle Force Deviation. Muscle force and EMG evolu-} tions of the lower limb muscle during the normal gait and the medial thrust gaits are shown in Figures 4 and 5. Overall, the comparisons revealed a fair qualitative agreement between muscle force and EMG-based patterns of each muscle during the normal gait and also medial thrust gait. The same observations were found for the bouncy, midcrouch, and mtp gait modifications. However, at some periods over the gait cycle, the high EMG activity does not perfectly correspond to the muscle peak force.
When analyzing the difference between each gait modification according to the normal gait, we observed altered muscle force ranges (Figure 6 and Table 3). Root mean square errors range from 7.9 to $139 \mathrm{~N}$, from 7.4 to $189.1 \mathrm{~N}$, from 5.7 to $101.3 \mathrm{~N}$, and from 7.4 to $126.2 \mathrm{~N}$ for the comparisons between the normal gait and the bouncy, medial thrust, midcrouch, and mtp gait modifications, respectively. Moreover, overall quantitative assessment revealed a root mean square error ranging from 5.5 to $189.1 \mathrm{~N}$ for all comparisons. The maximal deviation arises from the gluteus medius muscle during the medial thrust gait modification. The peroneus longus showed a minimal deviation during the midcrouch gait modification. Regarding the relative deviation, the overall range is from 10 to $34 \%$. The medial gastrocnemius revealed a minimal deviation during midcrouch gait modification while the sartorius showed a maximal deviation during the mtp gait modification.

3.3. Muscle Synergies. Muscle synergies extracted from the EMG signals during the midcrouch gait modifications are illustrated in Figure 7. We noted that the activation levels of the vastus lateralis and tibialis anterior are dominant for the midcrouch gait. The comparison between the muscle synergies patterns of the normal gait and other gait modifications is shown in Figure 8. We observed a large faction 

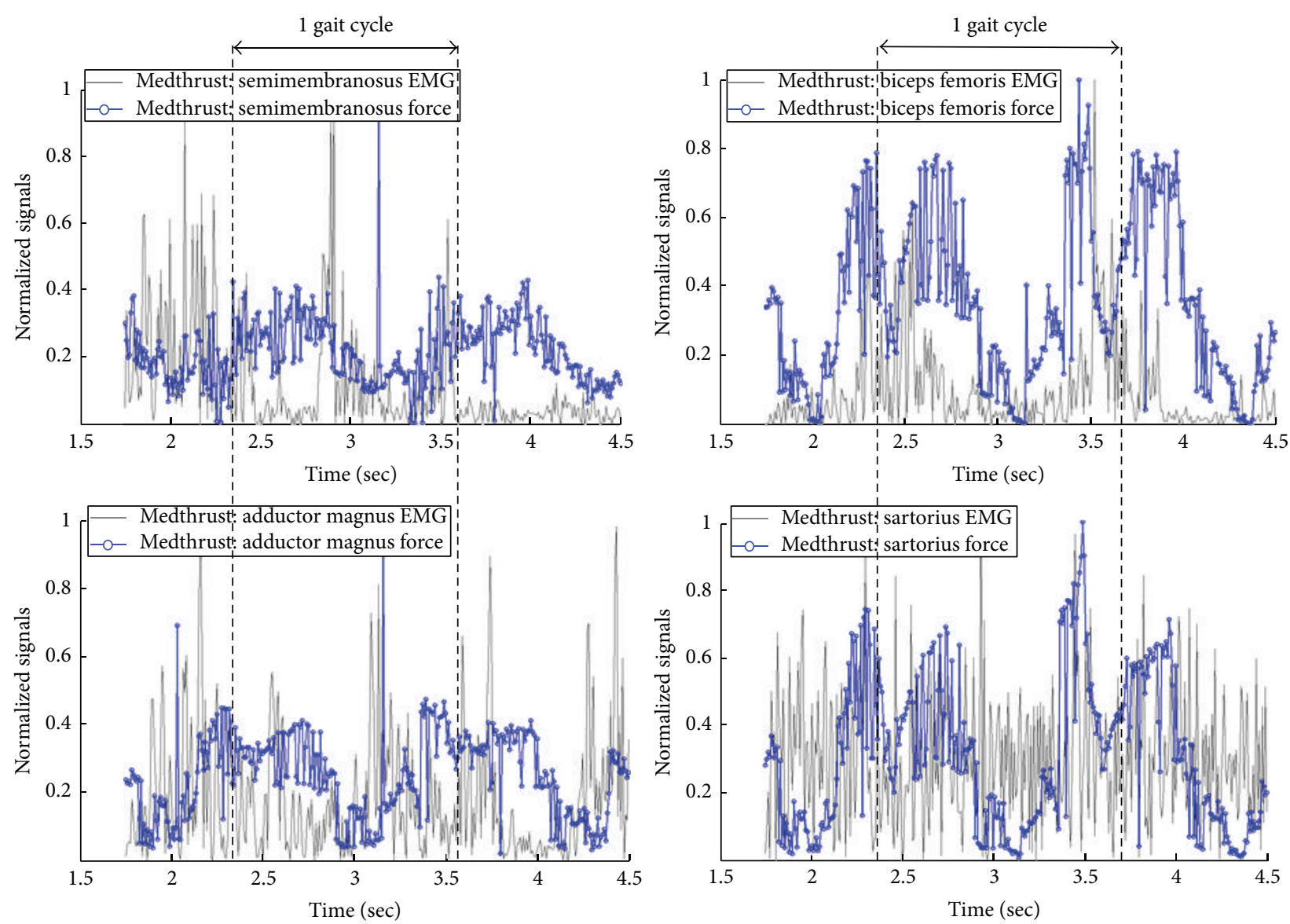

FIGURE 5: Lower limb muscle behaviors during the medial thrust gait: normalized tensile forces versus EMG signals.

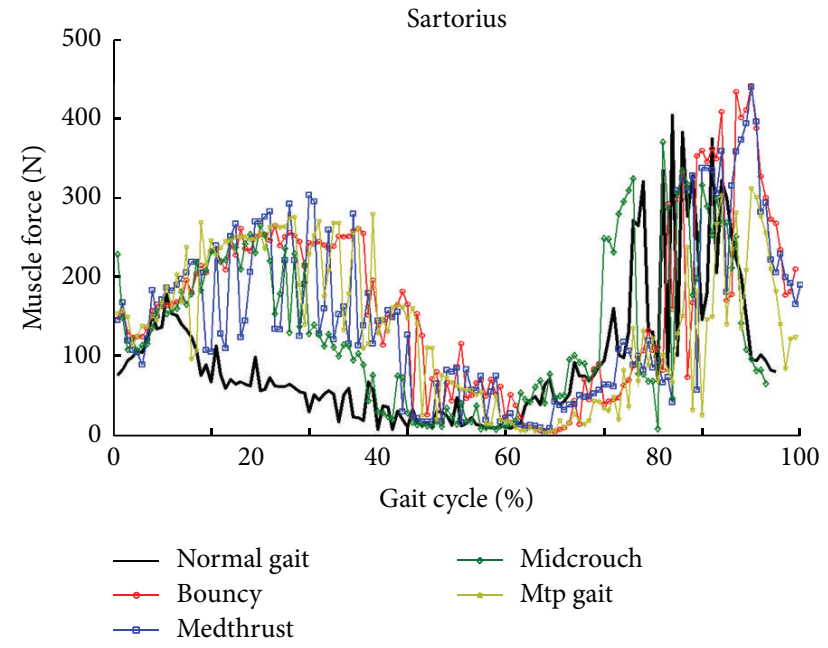

FIGURE 6: Evolution patterns of the thigh muscle (semimembranosus, biceps femoris, rectus femoris, and sartorius) forces: normal gait versus modification gait patterns.

of variation in the muscle patterns between the normal gait and all gait modifications. It is important to note that a small number of 4 synergies are sufficient to generate the functional output of muscle patterns. It is important to emphasize that one of the main objectives is to reach this number as small as possible when analyzing an important number of signals. Moreover, the activation level of the semimembranosus is more important for medial thrust and midcrouch gait modifications than other gaits.

\section{Discussion}

Neural motor control and its resulting joint kinematics and muscle forces provide basic knowledge of locomotion function of the human body in the normal as well as in the pathophysiological conditions [16, 33, 34]. Moreover, the better understanding of this complex underlying mechanism allows a better elucidation of the mechanisms behind body growth, aging progression, and disease development $[3,23]$. This may lead to a precise and accurate diagnosis of the musculoskeletal diseases. Furthermore, this provides also important knowledge in the fight (e.g., more efficient treatment prescription) against these musculoskeletal diseases. In this present study, the impact of the voluntary altered gait modifications on joint kinematics, muscle forces, and muscle synergies was assessed. Our findings contributed to the understanding of how the motor cortex changes voluntarily the motion patterns and its resulting effect on the joint and muscle mechanics. In fact, a large kinematic 


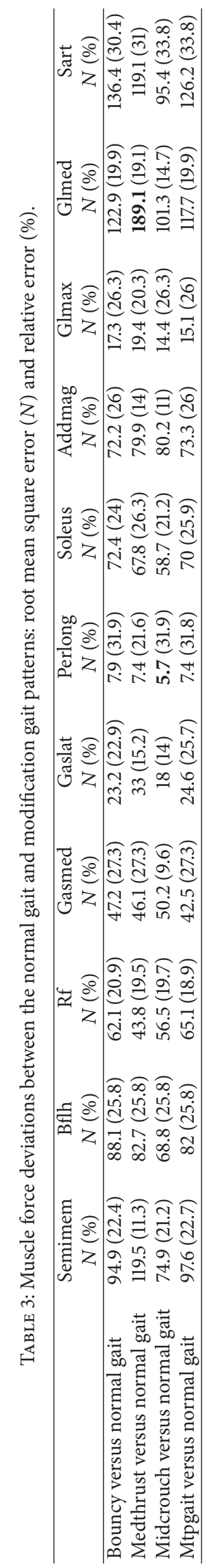



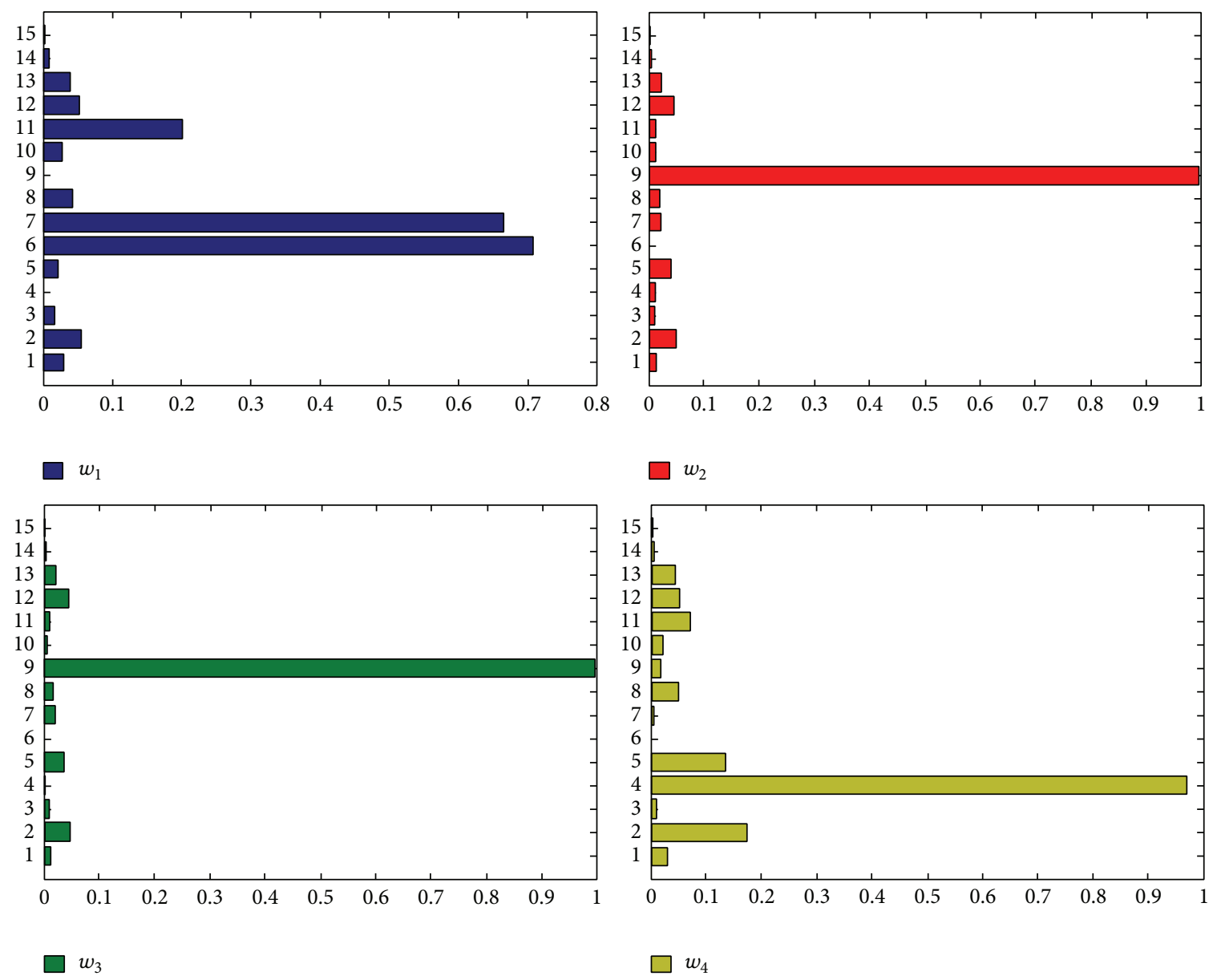

FIGURE 7: Muscle synergies matrix ( $w$-activation level) identified from the nonnegative matrix factorization based on the EMG signals collected during the midcrouch gait: (1) semimembranosus, (2) biceps femoris, (3) vastus medialis, (4) vastus lateralis, (5) rectus femoris, (6) medial gastrocnemius, (7) lateral gastrocnemius, (8) tensor fascia latae, (9) tibialis anterior, (10) peroneus longus, (11) soleus, (12) adductor magnus, (13) gluteus maximus, (14) gluteus medius, and (15) sartorius. Each column of muscle synergies $\left(w_{1}, w_{2}, w_{3}\right.$, and $\left.w_{4}\right)$ includes a vector of relative level of muscle activations of 15 analyzed muscles illustrated by horizontal bars.

deviation of around 19 degrees for knee flexion/extension was found between the normal gait and 4 other gait modifications (bouncy, medial thrust, midcrouch, and mtp). A significant change of $190 \mathrm{~N}$ for the lower limb muscle forces was also noted. In fact, our used musculoskeletal model was sensitive enough to detect the changes of the motor control strategies and their resulting effects on the joint and muscle behaviors. Motor commands may be generated through muscle synergy mechanism. Healthy human being is able to adapt a set of muscle synergies to effectively control body movements and stabilities. Nonetheless, abnormal gait (e.g., gait with instrumented implant in this present study) showed a large variation in the joint kinematics and muscle force patterns across a variety of gait modifications strategies. This could be explained by the fact that the implant may influence the decision-making process of the motor cortex leading to the joint and muscle adaptation behaviors, which are specific to each motion pattern.

In addition to the effect on the joint kinematics and muscle forces, EMG-based muscle synergy analysis revealed an important contribution of semimembranosus to the medial thrust and midcrouch gaits. Thus, this advanced multivariate analysis allows recognizing functional output of muscle activation patterns for each specific motion pattern [32]. In fact, we demonstrated that different altered motor behaviors may be constructed by a common set of muscle synergies (as results shown in Figures 7 and 8). Our findings support prior works related to the changes in the modular organization of different motion patterns of healthy and pathological subjects $[35,36]$.

At the moment, cutting-edge computational approaches such as inverse dynamics, static and dynamic optimization, and EMG-driven forward dynamics are commonly used to elucidate the neurophysiological relationships between the motor cortex, the muscle, and the joint mechanics [4]. Each approach has underlying advantages as well as disadvantages. Inverse dynamics and static optimization showed high residual results due to dynamic inconsistencies between ground reaction forces (GFR) and captured kinematics. Kinematical errors coming from anatomical representation, marker 

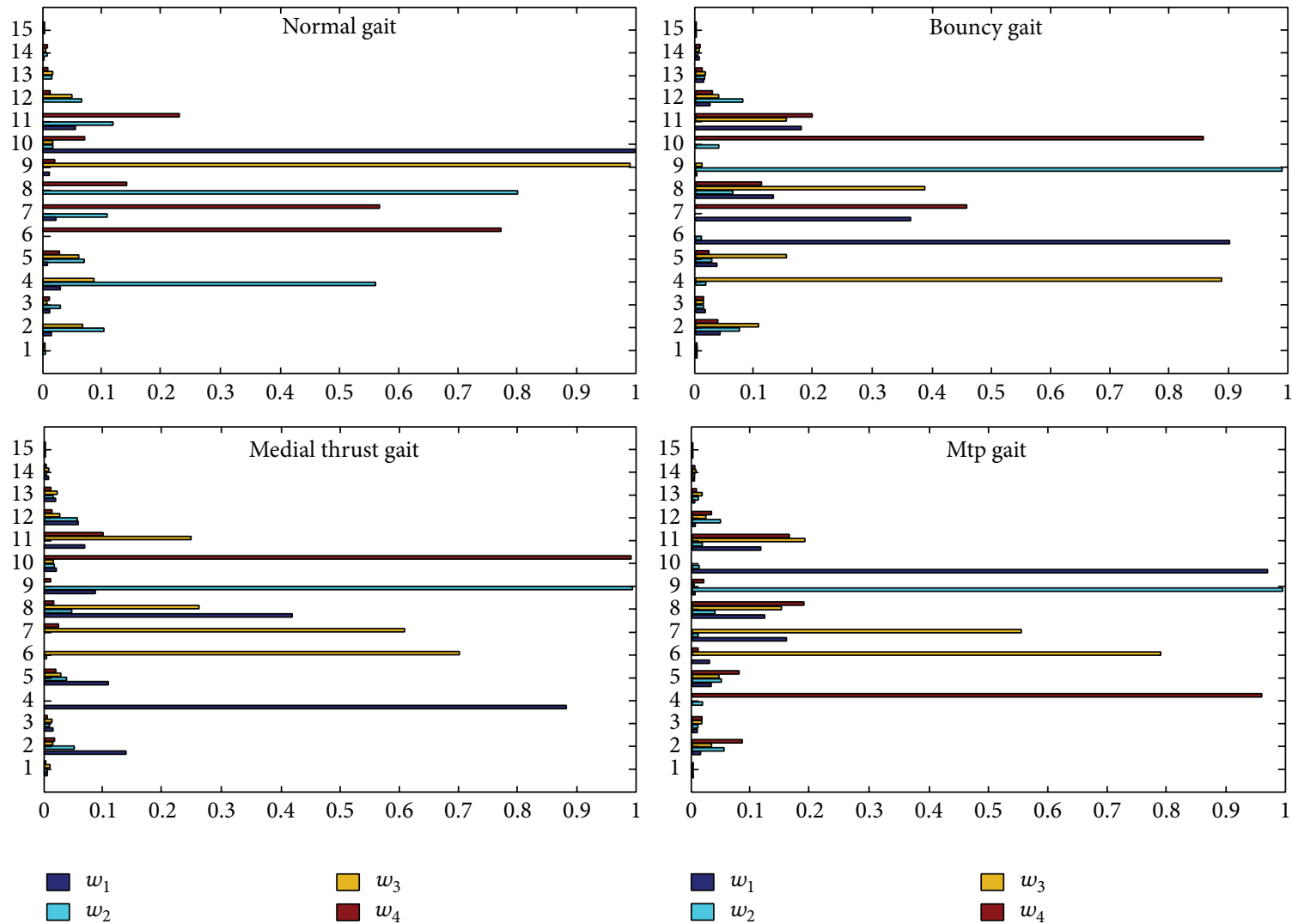

Figure 8: Comparison of all muscle synergies matrix ( $w$-activation level) between the normal gait and other gait modifications: (1) semimembranosus, (2) biceps femoris, (3) vastus medialis, (4) vastus lateralis, (5) rectus femoris, (6) medial gastrocnemius, (7) lateral gastrocnemius, (8) tensor fascia latae, (9) tibialis anterior, (10) peroneus longus, (11) soleus, (12) adductor magnus, (13) gluteus maximus, (14) gluteus medius, and (15) sartorius. Each gait pattern corresponds to the visualization of all 15 muscles of interest and their associated synergies. Each column of muscle synergies $\left(w_{1}, w_{2}, w_{3}\right.$, and $\left.w_{4}\right)$ includes a vector of relative level of muscle activations of 15 analyzed muscles illustrated by horizontal bars.

registration, and soft tissue artifacts may alter the accuracy of the results [16]. Moreover, the inaccuracy may come from the noise in GRF or drift effect over time. Furthermore, the choice of the objective function may create nonuniqueness solutions. However, this approach has a short computing time and this may be appropriately suitable for real-time simulation. Forward dynamics and dynamic optimization have computational complexity. The accuracy of EMG signals due to electrode location and signal processing approach may alter significantly the simulation results [37]. Another challenge is how to deal with the recruitment mechanism of related motor units in pathological muscles. Thus, the estimation of muscle forces using optimization deals with nonuniqueness cost function and this approach may be invalid for movement disorders. The EMG-driven approach is challenging, but there are many disadvantageous factors related to the cross talk effect and noise or uncertain muscle model parameters. Moreover, it is especially difficult to acquire accurately deep muscle excitations in noninvasive manner. In fact, the choice of the appropriate approaches depends on the specific objective of study. In this present study, inverse dynamics and static optimization were applied to estimate the lower limb muscle forces. The comparisons between muscle force patterns and EMG-based activation patterns showed a good agreement (Figures 4 and 5) for the normal gait pattern. This finding confirmed the reasonable accuracy of the used approach in the estimation of in vivo patient-specific healthy muscle forces $[6,20]$. However, it is important to emphasize that the use of EMG signals allows only a qualitative validation of the estimated muscle forces to be achieved. Consequently, the present study showed some disagreements between estimated muscle forces and EMG signals, especially for modified gait patterns. This phenomenon could be explained by the fact that the modeling of abnormal muscle behaviors, especially with instrumented implant devices, remains a challenging issue. Moreover, the use of an activation-based optimization function may be not well appropriate for these modified gaits. In perspective, further works related to the definition of new optimization functions for abnormal gaits need to be performed to improve the simulation outcomes. Furthermore, electrical activities of the semimembranosus and adductor magnus muscles do not match the EMG profiles of the healthy subjects [38]. The differences may be explained by the fact that the instrumented implant changed the muscle behaviors. In fact, more works are needed to elucidate this point. 
The medial thrust gait allows the footpath to be changed during gait cycle. Recent study showed decreases of knee adduction torque $(-34 \%)$ and hip abduction torque $(-15 \%)$ by using this gait pattern [13]. Another study [39] showed the increases of knee contact forces by $23 \%, 29 \%$, and $36 \%$ in the lateral side using mild crouch, moderate crouch, and bouncy gait modifications, respectively. Despite their inexpensive character for the disease management, specific gait patterns such as crouch or bouncy and especially the medial knee thrust gait are quite complex motion patterns. At the moment, there is no universal method due to the lack of long-term effectiveness study. The challenges related to these gait-retaining strategies remain in the determination of optimal strategy and magnitude of modifications required to maximize beneficial effects and an efficient and special training method with real-time biofeedback is needed [14]. Muscle synergies analysis based on the dimensional reduction principle (i.e., matrix factorization) allows studying how the nervous system reduces movement control complexity [40]. In this present study, the muscle synergy analysis provides muscle activation pattern of each specific muscle related to each gait modification (bouncy, medial thrust, mild crouch, and mtp) leading to determining its effect on the muscle biomechanics. Moreover, our findings are in agreement with the literature works showing that only 37 neural commands (i.e., number of muscle synergies) are required for walking activities [39].

The use of a single subject with instrumented implant is the main limitation of this study. Consequently, more subjects are needed to generalize our findings. However, this present study provided unique and important information about the effect of voluntary gait modification strategies on the muscle biomechanics (e.g., activations and forces). The validation of the musculoskeletal simulation results is also a current challenging issue. This modeling problem depends upon objective and context of the research question and how modeling hypotheses are performed. At the moment, new experimental technologies and protocols need to be developed to provide new exploitable data for validation and verification purpose. In the scope of this objective, a Knee Grand Challenge has been organized to validate the joint loading and muscle forces by using joint reaction forces measured from instrumented implants. This provides a large panel of experimental data for musculoskeletal modeling purposes. In this present study, muscle force patterns were provided for the normal gait and 4 gait modifications. Then, EMG-based activation patterns were compared to muscle force patterns to evaluate the accuracy of the developed model. These data will be used to validate the joint reaction forces by a new approach of validation based on musculoskeletal simulation results and machine learning technique.

\section{Conclusions}

This present study showed an important variation of kinematical and muscle force behaviors under the voluntary altered gait patterns. Our findings allowed also muscle synergies to be recognized for the normal gait as well as 4 gait modifications (bouncy, medial thrust, midcrouch, and mtp). In fact, such useful information could allow a better understanding of the joint function and muscle synergy strategies leading to deeper knowledge of joint and muscle mechanisms related to neural commands.

\section{Highlights}

(i) Effect of voluntary gait modifications on the human knee joint behaviors.

(ii) Impact of structure-modifying treatment strategies on the muscle behaviors.

(iii) Lower limb muscle synergy analysis using nonnegative matrix factorization.

(iv) Patient-specific musculoskeletal modeling and simulation.

\section{Conflict of Interests}

The author reports no conflict of interests.

\section{Acknowledgment}

The author would like to thank all the organizers of the Knee Grand Challenge for the available data used in this present study.

\section{References}

[1] S. J. Piazza and S. L. Delp, "The influence of muscles on knee flexion during the swing phase of gait," Journal of Biomechanics, vol. 29, no. 6, pp. 723-733, 1996.

[2] M. G. Pandy, "Computer modeling and simulation of human movement," Annual Review of Biomedical Engineering, vol. 3, pp. 245-273, 2001.

[3] T. S. Buchanan, D. G. Lloyd, K. Manal, and T. F. Besier, "Neuromusculoskeletal modeling: estimation of muscle forces and joint moments and movements from measurements of neural command," Journal of Applied Biomechanics, vol. 20, no. 4, pp. 367-395, 2004.

[4] A. Erdemir, S. McLean, W. Herzog, and A. J. van den Bogert, "Model-based estimation of muscle forces exerted during movements," Clinical Biomechanics, vol. 22, no. 2, pp. 131-154, 2007.

[5] E. M. Arnold, S. R. Hamner, A. Seth, M. Millard, and S. L. Delp, "How muscle fiber lengths and velocities affect muscle force generation as humans walk and run at different speeds," Journal of Experimental Biology, vol. 216, no. 11, pp. 2150-2160, 2013.

[6] T. T. Dao, P. Pouletaut, F. Charleux et al., "Estimation of patient specific lumbar spine muscle forces using multi-physical musculoskeletal model and dynamic MRI," Advances in Intelligent and Soft Computing, vol. 245, pp. 411-422, 2014.

[7] A. S. Arnold, M. Q. Liu, M. H. Schwartz, S. Õunpuu, L. S. Dias, and S. L. Delp, "Do the hamstrings operate at increased muscletendon lengths and velocities after surgical lengthening?" Journal of Biomechanics, vol. 39, no. 8, pp. 1498-1506, 2006. 
[8] J. L. Hicks, S. L. Delp, and M. H. Schwartz, "Can biomechanical variables predict improvement in crouch gait?" Gait and Posture, vol. 34, no. 2, pp. 197-201, 2011.

[9] P. Gerus, M. Sartori, T. F. Besier et al., "Subject-specific knee joint geometry improves predictions of medial tibiofemoral contact forces," Journal of Biomechanics, vol. 46, no. 16, pp. 2778-2786, 2013.

[10] H. J. Kim, J. W. Fernandez, M. Akbarshahi, J. P. Walter, B. J. Fregly, and M. G. Pandy, "Evaluation of predicted knee-joint muscle forces during gait using an instrumented knee implant," Journal of Orthopaedic Research, vol. 27, no. 10, pp. 1326-1331, 2009.

[11] M. W. Hast and S. J. Piazza, "Dual-joint modeling for estimation of total knee replacement contact forces during locomotion," Journal of Biomechanical Engineering, vol. 135, no. 2, Article ID 021013, 9 pages, 2013.

[12] B. J. Fregly, J. A. Reinbolt, K. L. Rooney, K. H. Mitchell, and T. L. Chmielewski, "Design of patient-specific gait modifications for knee osteoarthritis rhabilitation," IEEE Transactions on Biomedical Engineering, vol. 54, no. 9, pp. 1687-1695, 2007.

[13] B. Fregly, "Computational assessment of combinations of gait modifications for knee osteoarthritis rehabilitation," IEEE Transactions on Biomedical Engineering, vol. 55, no. 8, pp. 21042106, 2008.

[14] B. J. Fregly, "Gait modification to treat knee osteoarthritis," HSS Journal, vol. 8, no. 1, pp. 45-48, 2012.

[15] M. Simic, R. S. Hinman, T. V. Wrigley, K. L. Bennell, and M. A. Hunt, "Gait modification strategies for altering medial knee joint load: a systematic review," Arthritis Care and Research, vol. 63, no. 3, pp. 405-426, 2011.

[16] T. T. Dao, F. Marin, P. Pouletaut, F. Charleux, P. Aufaure, and M. C. Ho Ba Tho, "Estimation of accuracy of patientspecific musculoskeletal modelling: case study on a post polio residual paralysis subject," Computer Methods in Biomechanics and Biomedical Engineering, vol. 15, no. 7, pp. 745-751, 2012.

[17] K. Manal and T. S. Buchanan, "An electromyogram-driven musculoskeletal model of the knee to predict in vivo joint contact forces during normal and novel gait patterns," Journal of Biomechanical Engineering, vol. 135, no. 2, Article ID 021007, 2013.

[18] D. T. Davy and M. L. Audu, "A dynamic optimization technique for predicting muscle forces in the swing phase of gait," Journal of Biomechanics, vol. 20, no. 2, pp. 187-201, 1987.

[19] F. C. Anderson and M. G. Pandy, "Dynamic optimization of human walking," Journal of Biomechanical Engineering, vol. 123, no. 5, pp. 381-390, 2001.

[20] F. C. Anderson and M. G. Pandy, "Static and dynamic optimization solutions for gait are practically equivalent," Journal of Biomechanics, vol. 34, no. 2, pp. 153-161, 2001.

[21] M. S. A. Graziano, C. S. R. Taylor, and T. Moore, "Complex movements evoked by microstimulation of precentral cortex," Neuron, vol. 34, no. 5, pp. 841-851, 2002.

[22] I. Stepniewska, P.-C. Fang, and J. H. Kaas, "Microstimulation reveals specialized subregions for different complex movements in posterior parietal cortex of prosimian galagos," Proceedings of the National Academy of Sciences of the United States of America, vol. 102, no. 13, pp. 4878-4883, 2005.
[23] F. O. Barroso, D. Torricelli, J. C. Moreno et al., "Shared muscle synergies in human walking and cycling," Journal of Neurophysiology, vol. 112, no. 8, pp. 1984-1998, 2014.

[24] B. J. Fregly, T. F. Besier, D. G. Lloyd et al., "Grand challenge competition to predict in vivo knee loads," Journal of Orthopaedic Research, vol. 30, no. 4, pp. 503-513, 2012.

[25] S. L. Delp, F. C. Anderson, A. S. Arnold et al., "OpenSim: opensource software to create and analyze dynamic simulations of movement," IEEE Transactions on Biomedical Engineering, vol. 54, no. 11, pp. 1940-1950, 2007.

[26] G. Wu, S. Siegler, P. Allard et al., "ISB recommendation on definitions of joint coordinate system of various joints for the reporting of human joint motion-part I: ankle, hip, and spine," Journal of Biomechanics, vol. 35, no. 4, pp. 543-548, 2002.

[27] E. M. Arnold, S. R. Ward, R. L. Lieber, and S. L. Delp, "A model of the lower limb for analysis of human movement," Annals of Biomedical Engineering, vol. 38, no. 2, pp. 269-279, 2010.

[28] L. M. Schutte, M. Rodgers, F. E. Zajac, and R. M. Glaser, "Improving the efficacy of electrical stimulation-induced leg cycle ergometry: an analysis based on a dynamic musculoskeletal model," IEEE Transactions on Rehabilitation Engineering, vol. 1, no. 2, pp. 109-125, 1993.

[29] P. Paatero and U. Tapper, "Positive matrix factorization: a nonnegative factor model with optimal utilization of error estimates of data values," Environmetrics, vol. 5, no. 2, pp. 111-126, 1994.

[30] D. D. Lee and H. S. Seung, "Learning the parts of objects by non-negative matrix factorization," Nature, vol. 401, no. 6755, pp. 788-791, 1999.

[31] J. Kim and H. Park, "Fast nonnegative matrix factorization: an active-set-like method and comparisons," SIAM Journal on Scientific Computing, vol. 33, no. 6, pp. 3261-3281, 2011.

[32] D. J. Berger and A. d'Avella, "Effective force control by muscle synergies," Frontiers in Computational Neuroscience, vol. 8, no. 1, article 46, 2014.

[33] A. S. Arnold, M. Q. Liu, M. H. Schwartz, S. Õunpuu, L. S. Dias, and S. L. Delp, "Do the hamstrings operate at increased muscletendon lengths and velocities after surgical lengthening?" Journal of Biomechanics, vol. 39, no. 8, pp. 1498-1506, 2006.

[34] H. J. Lundberg, C. Knowlton, and M. A. Wimmer, "Fine tuning total knee replacement contact force prediction algorithms using blinded model validation," Journal of Biomechanical Engineering, vol. 135, no. 2, Article ID 021008, 2013.

[35] L. Gizzi, J. F. Nielsen, F. Felici, Y. P. Ivanenko, and D. Farina, "Impulses of activation but not motor modules are preserved in the locomotion of subacute stroke patients," Journal of Neurophysiology, vol. 106, no. 1, pp. 202-210, 2011.

[36] K. L. Rodriguez, R. T. Roemmich, B. Cam, B. J. Fregly, and C. J. Hass, "Persons with Parkinson's disease exhibit decreased neuromuscular complexity during gait," Clinical Neurophysiology, vol. 124, no. 7, pp. 1390-1397, 2013.

[37] D. Staudenmann, K. Roeleveld, D. F. Stegeman, and J. H. van Dieen, "Methodological aspects of SEMG recordings for force estimation-a tutorial and review," Journal of Electromyography and Kinesiology, vol. 20, no. 3, pp. 375-387, 2010.

[38] J. Perry and J. M. Burnfield, Gait Analysis: Normal and Pathological Function, SLACK Incorporated, Thorofare, NJ, USA, 2010. 
[39] A. L. Kinney, T. F. Besier, A. Silder, S. L. Delp, D. D. D’Lima, and B. J. Fregly, "Changes in in vivo knee contact forces through gait modification," Journal of Orthopaedic Research, vol. 31, no. 3, pp. 434-440, 2013.

[40] J. P. Walter, A. L. Kinney, S. A. Banks et al., "Muscle synergies may improve optimization prediction of knee contact forces during walking," Journal of Biomechanical Engineering, vol. 136, no. 2, Article ID 021031, 2014. 


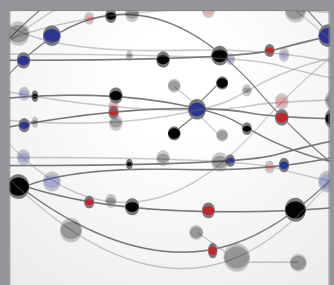

The Scientific World Journal
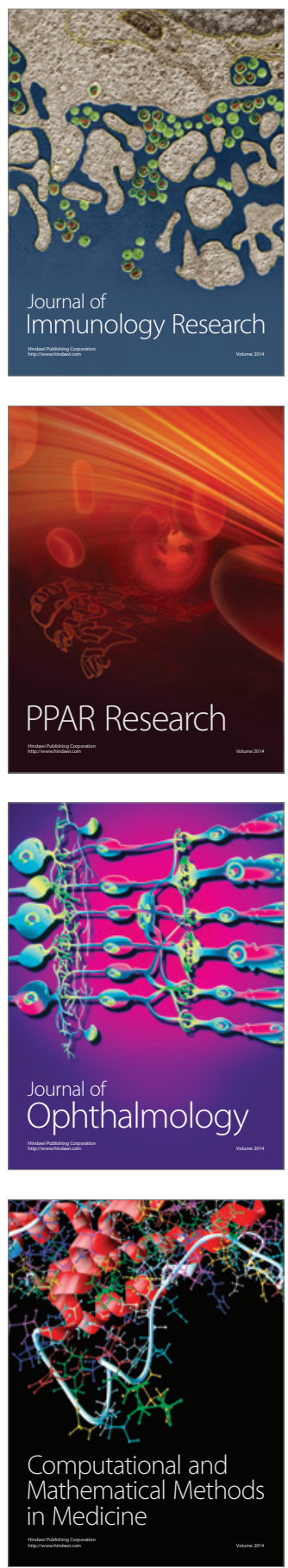

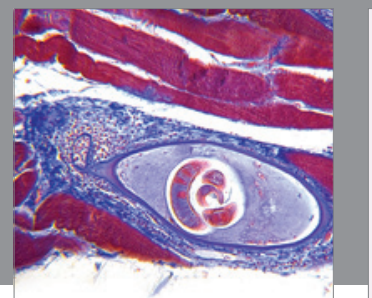

Gastroenterology

Research and Practice
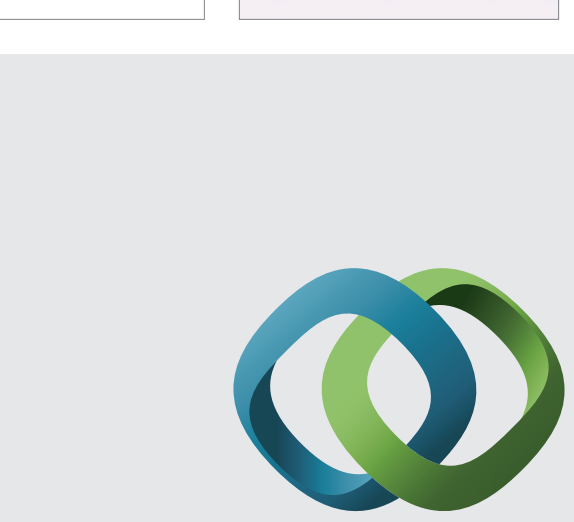

\section{Hindawi}

Submit your manuscripts at

http://www.hindawi.com
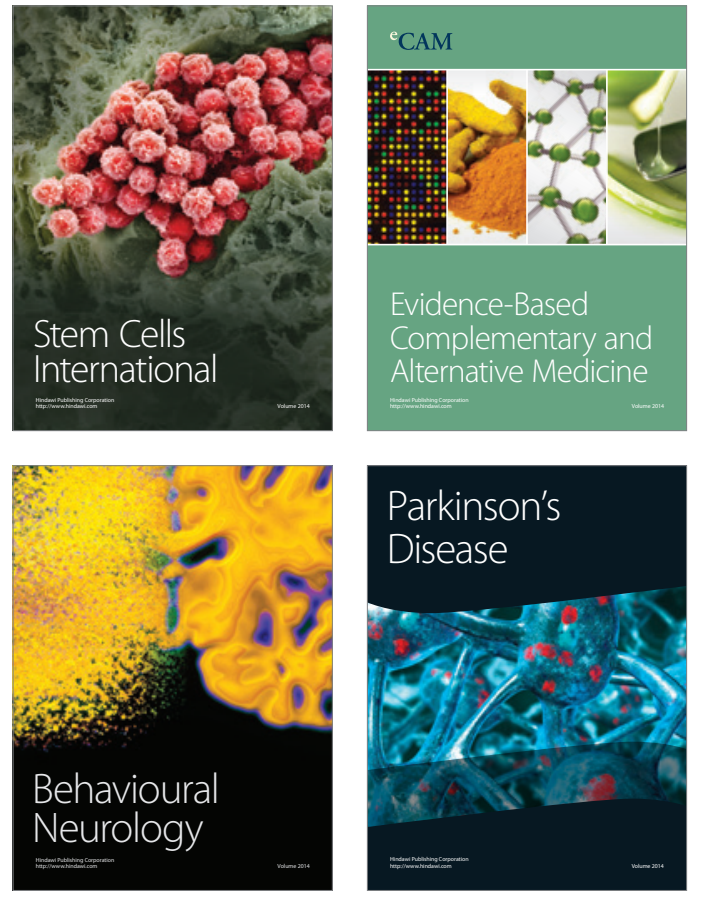
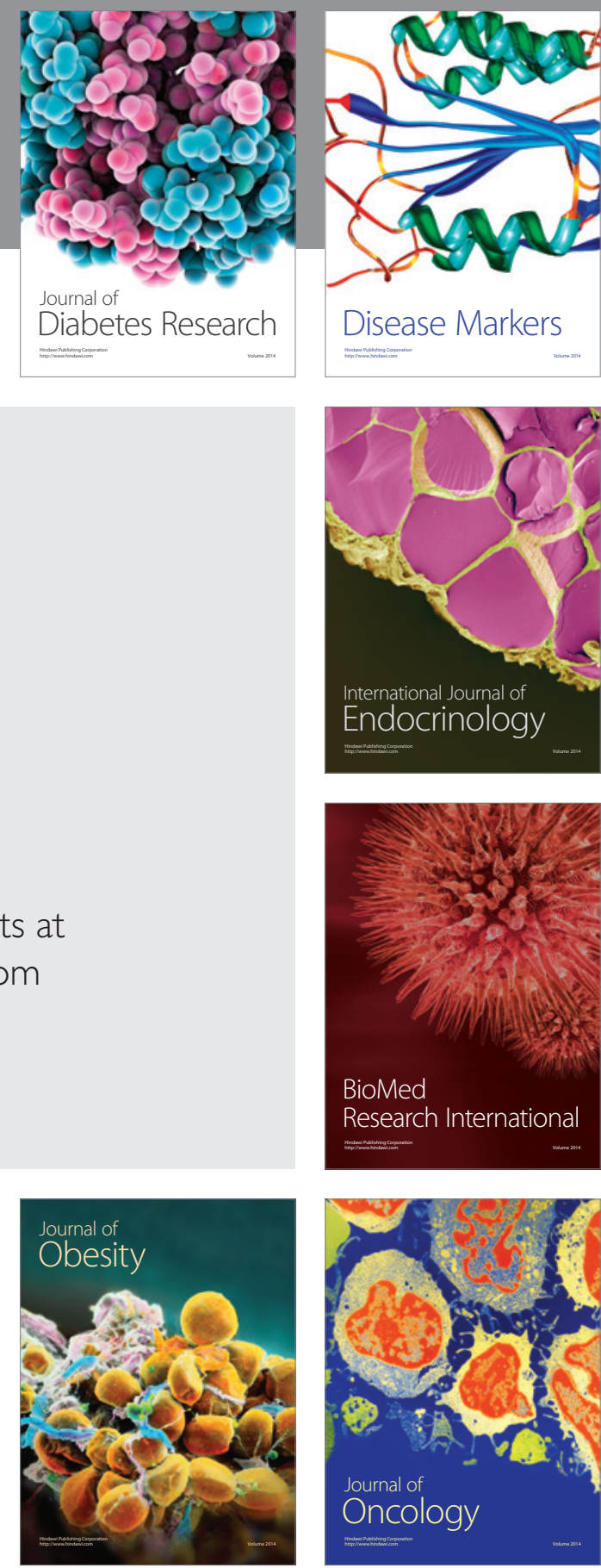

Disease Markers
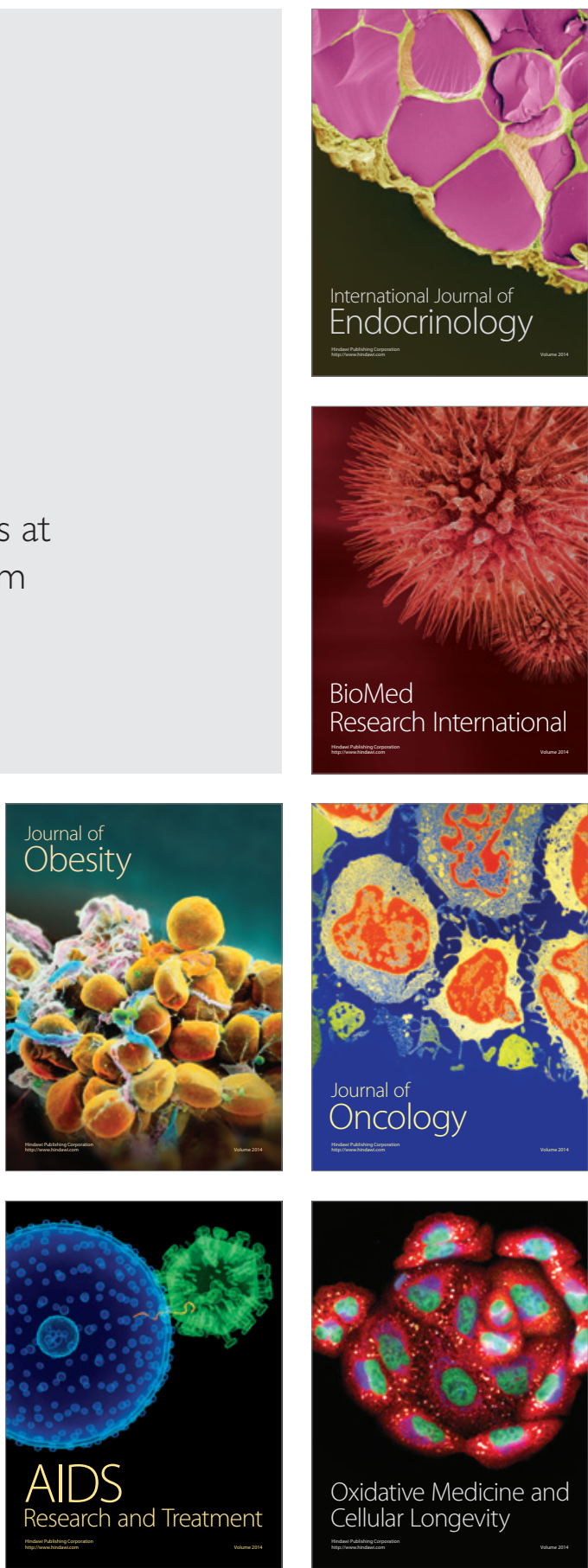